\title{
Nivel de Educación Parental y Mortalidad Infantil
}

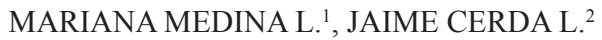 \\ 1. Departamento de Pediatría, Facultad de Medicina, Pontificia Universidad Católica de Chile. \\ 2. Departamento de Salud Pública, Facultad de Medicina, Pontificia Universidad Católica de Chile.
}

\begin{abstract}
Interaction between maternal and paternal educational level as conditionant of infant mortality

Background: In the study of the factors that affect infant mortality the interaction of variables has been poorly explored. Objective: Exemplify a method to assess interaction between two variables associated to infant mortality (maternal and paternal educational level). Materials and Methods: Based on data published in the Demography Yearbook (2007), infant mortality rate (IMR) was calculated for 9 combinations of maternal and paternal years of schooling $(0-9,10-12$ y $13+$ years $)$ and the IMR ratio between combinations. Interaction was evaluated by comparing the sum of the attributable risk of discordant combinations (0-9 years of schooling mother and $13+$ years of schooling father, and vice versa) versus the combination (13+ years of schooling mother and father). Results: IMR of the combination ( $0-9$ years of schooling mother and father) was 2.7 fold the combination (13+ years of schooling mother and father), being also greater than the sum of the attributable risk of the discordant combinations. Conclusion: The existence of a synergic interaction in additive scale between maternal and paternal education level was demonstrated. Identifying the presence of interactions leads to a focalized implementation of sanitary policies, raising their effectiveness.

(Key words: Educational level, infant mortality, interaction).

Rev Chil Pediatr 2010; 81 (3): 228-233
\end{abstract}

\section{RESUMEN}

Antecedentes: En el estudio de los factores condicionantes de mortalidad infantil la interacción de variables constituye un fenómeno poco explorado. Objetivo: Ejemplificar una forma de abordaje del fenómeno de interacción entre dos variables asociadas a mortalidad infantil (nivel educacional materno y paterno). Material y Métodos: En base a los datos publicados en el Anuario de Demografía (2007) se calculó la tasa de mortalidad infantil (TMI) para 9 combinaciones de años de estudios aprobados (AEA) de la madre y del padre (0-9, 10-12 y $13+$ años), y la razón de TMI entre las combinaciones. La evaluación de interacción se realizó comparando la suma del riesgo atribuible de las combinaciones discordantes (0-9 AEA madre y 13+ AEA padre, y viceversa) versus la combinación (13+ AEA madre y padre). Resultados: La TMI de la combinación (0-9 AEA madre

Trabajo recibido el 17 de febrero de 2010, devuelto para corregir el 15 de marzo de 2010, segunda versión el 05 de abril de 2010, aceptado para publicación el 08 de abril de 2010.

Correspondencia a:

Dr. Jaime Cerda L.

E-mail.: jcerda@med.puc.cl 
y padre) fue 2,7 veces mayor a la combinación (13+ AEA madre y padre); siendo también mayor la suma del riesgo atribuible de las combinaciones discordantes. Conclusión: Se demostró la existencia de una interacción en escala aditiva de tipo sinérgico entre el nivel educacional materno y paterno. Identificar la presencia de interacciones permite focalizar políticas sanitarias, aumentando su efectividad.

(Palabras clave: Nivel educacional, mortalidad infantil, interacción).

Rev Chil Pediatr 2010; 81 (3): 228-233

\section{Introducción}

La disminución sostenida de la mortalidad infantil ocurrida en Chile durante la segunda mitad del siglo XX ha sido objeto de estudio a nivel mundial, por tratarse del singular caso de un país en vías de desarrollo cuya tasa descendió desde 136,2/1 000 nacidos vivos (1950) a $8,9 / 1000$ nacidos vivos (2000), a una velocidad promedio de $-2,5$ puntos porcentuales/ año ${ }^{1}$. Hoy en día, Chile presenta una tasa de mortalidad infantil similar a la reportada por países del primer mundo, concentrando sus esfuerzos en la resolución de nuevos problemas de salud característicos de países en fases avanzadas de transición demográfica y epidemiológica (e.g. patologías crónicas del adulto, obesidad infantil). Ante este nuevo escenario, pareciera ser que el estudio de los factores condicionantes de mortalidad infantil es materia del pasado. Tal aseveración dista de la realidad, toda vez que la tasa de mortalidad infantil de nuestro país aún presenta un margen de reducción, existen importantes desigualdades en la mortalidad infantil entre distintos estamentos de nuestra sociedad ${ }^{2-4} \mathrm{y}$, por cierto, el fenómeno aún no ha sido comprendido a cabalidad, existiendo numerosos factores por conocer, así como otros cuya comprensión es aún parcial (e.g. determinantes sociales). Conciente de esta realidad, la autoridad sanitaria incluyó entre los Objetivos Sanitarios para la Década 2000-2010 reducir en 25\% la mortalidad infantil a nivel nacional y en $10 \%$ la brecha entre quintiles extremos de escolaridad materna (cuyo riesgo relativo fue 2,6 en el trienio 1998-2000) .

Históricamente el estudio de los factores de riesgo de muerte del menor de 1 año ha sido realizado en base al análisis de cada variable en forma aislada ${ }^{6}$. Nuevas metodologías de análisis permiten en la actualidad profundizar el conocimiento vigente, principalmente al reducir la presencia de confusión. Sin embargo, un elemento muy poco explorado ha sido el fenómeno de interacción de variables, también conocido como modificación de efecto. Desde un punto de vista biológico, se habla de interacción entre dos o más factores causales cuando existe una interdependencia entre sus mecanismos de acción para causar, prevenir o controlar una enfermedad, de manera que la incidencia de enfermedad atribuible a este conjunto de factores causales es diferente de la incidencia esperable de sus efectos individuales $^{7}$. El conocimiento sobre interacciones causales no es solamente de interés académico, por el contrario, tiene importantes implicancias en el ámbito de la salud pública, ya que al identificar grupos o escenarios en los cuales ocurren interacciones, las acciones preventivas pueden ser más efectivas ${ }^{8}$.

La exploración del fenómeno de interacción en un problema de salud pública pediátrico como es la mortalidad infantil motivó la realización del presente estudio, siendo sus objetivos (i) ejemplificar una forma de abordaje del fenómeno de interacción entre dos variables asociadas a mortalidad infantil y (ii) profundizar el conocimiento vigente sobre la forma en la que el nivel educacional de ambos progenitores condiciona la muerte del menor de 1 año.

\section{Pacientes y Método}

Datos sobre defunciones de menores de 1 año y número de nacidos vivos, desagregados según años de estudios aprobados (AEA) de la madre y del padre, se obtuvieron a partir del Anuario de Estadísticas Vitales (2007)9. 
Los AEA fueron estratificados en tres niveles: 0-9 años, 10-12 años y 13+ años; originando 9 combinaciones de AEA maternos y paternos. Para cada una de las combinaciones se calculó la tasa de mortalidad infantil (TMI), definida según la fórmula (muertes $<1$ año/1 000 nacidos vivos). Como una forma de simplificar la presentación de la metodología y resultados se utilizó la siguiente clave: letras $\mathrm{m}$ y $\mathrm{M}=$ madre, letras $\mathrm{p}$ y $\mathrm{P}=$ padre; letras minúsculas =0-9 AEA, letras mayúsculas $=13+$ AEA. Por ejemplo, la pareja $m P$ corresponde a una madre con 0-9 AEA y un padre con 13+ AEA.

Se calculó la razón de tasas de mortalidad infantil (RT) entre cada combinación de AEA maternos y paternos versus la combinación de referencia $M P$. Para evaluar el fenómeno de interacción entre los AEA maternos y paternos se calculó el riesgo atribuible (RA) de tres combinaciones, a saber, $m p, m P$ y $M p$. El riesgo atribuible se calculó como la diferencia de la razón de tasas de cada una de las combinaciones y el riesgo basal, correspondiente al riesgo atribuible a la combinación $M P$. La presencia de una interacción en escala aditiva se verificó en caso de no cumplirse la siguiente igualdad: $(\mathrm{RA} m p)=(\mathrm{RA} m P+\mathrm{RA} M p)^{10}$.

\section{Resultados}

Durante el año 2007 se registraron un total de 240539 nacidos vivos y 2009 defunciones de menores de 1 año (TMI $=8,4 / 1000$ nacidos vivos), de los cuales $213035(88,6 \%)$ nacidos vivos y $1726(85,9 \%)$ defunciones de menores de 1 año contaban con información sobre los AEA de ambos progenitores. La tabla 1 resume la TMI de cada combinación de AEA maternos y paternos. Los valores marginales muestran que, independientemente de los AEA paternos, la TMI desciende a medida que aumentan los AEA maternos; por su parte, independientemente de los AEA maternos, la TMI desciende a medida que aumentan los AEA paternos. La combinación $M P$ presentó la menor TMI (4,93/1 000 nacidos vivos); por su parte, la combinación $m p$ presentó la mayor TMI (13,07/1 000 nacidos vivos). Las combinaciones discordantes (i.e. $m P$ y $M p$ ) presentaron TMI de 5,96 y 5,33/1 000 nacidos vivos, respectivamente.

La tabla 2 resume la razón de TMI entre cada combinación de AEA maternos y paternos versus la combinación de referencia. $\mathrm{La}$ razón de TMI tomó su valor máximo para la combinación $m p$, siendo ésta 2,7 veces el valor de la tasa de referencia (MP). Las combinaciones discordantes de AEA entre madres y padres también presentaron una mayor razón de TMI comparadas con la combinación de referencia (RT $M p=1,08$ y RT $m P=1,21$ ).

Por último, el análisis de categorías extremas de AEA revela la existencia de una interacción aditiva entre los AEA materno y paterno, por cuanto el riesgo atribuible a la combinación $m p(2,65-1,00=1,65)$ es diferente a la sumatoria del riesgo atribuible de las combinaciones $m P(1,21-1,00=0,21)$ y $M p(1,08-1,00$ $=0,08)$. Al cumplirse que (RA $m p>$ RA $m P+$ RA $M p)$, la interacción aditiva recibe el calificativo de sinérgico (figura 1).

Tabla 1. Tasa de mortalidad infantil (1/1 000), según años de estudios aprobados por la madre y el padre (Chile, 2007)

\begin{tabular}{|c|c|c|c|c|c|}
\hline \multirow{2}{*}{\multicolumn{2}{|c|}{ Años de estudios aprobados }} & \multicolumn{4}{|c|}{ Padre } \\
\hline & & $0-9$ & $10-12$ & $13+$ & Total \\
\hline \multirow{4}{*}{ Madre } & $0-9$ & 13,07 & 10,05 & 5,96 & 11,58 \\
\hline & $10-12$ & 8,79 & 8,19 & 6,90 & 8,07 \\
\hline & $13+$ & 5,33 & 7,83 & 4,93 & 5,54 \\
\hline & Total & 11,02 & 8,47 & 5,52 & 8,10 \\
\hline
\end{tabular}

$\mathrm{TMI}=$ muertes $<1$ año/1 000 nacidos vivos. 
Tabla 2. Razón de tasas de mortalidad infantil, según años de estudios aprobados por la madre y el padre (Chile, 2007)

\begin{tabular}{|clccc|}
\hline Años de estudios aprobados & & Padre & \\
& & $\mathbf{0 - 9}$ & $\mathbf{1 0 - 1 2}$ & $\mathbf{1 3 +}$ \\
Madre & $\mathbf{0 - 9}$ & 2,65 & 2,04 & 1,21 \\
& $\mathbf{1 0 - 1 2}$ & 1,78 & 1,66 & 1,40 \\
& $\mathbf{1 3 +}$ & 1,08 & 1,59 & 1,00 \\
\hline
\end{tabular}

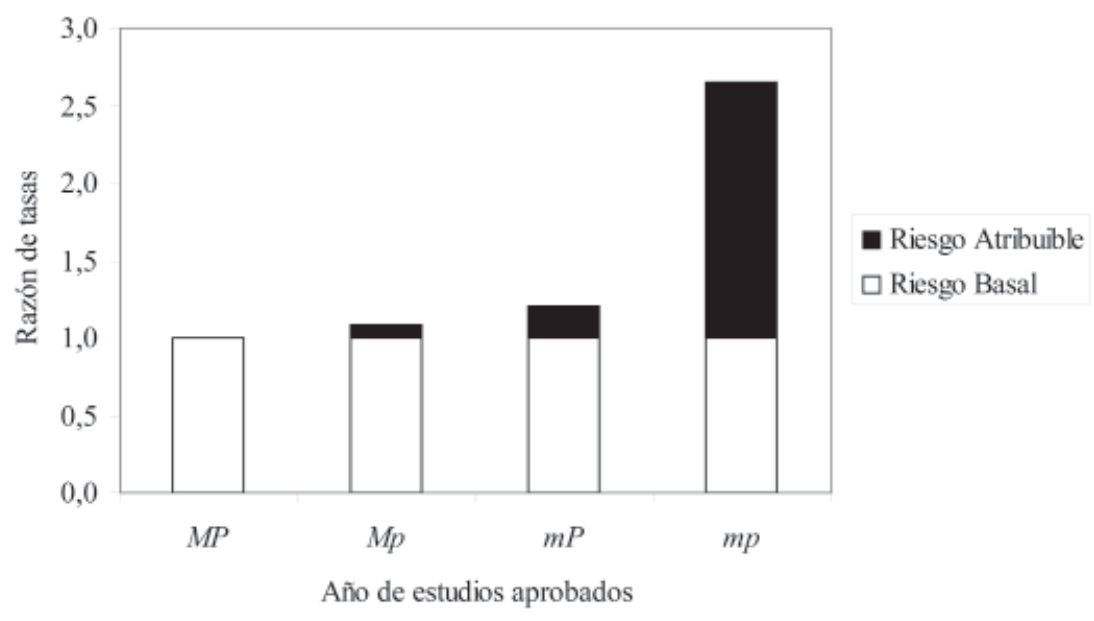

MP: Años de estudios aprobados $=13+($ madre $)$ y $13+$ (padre) Mp: Años de estudios aprobados $=13+$ (madre) y $0-9$ (padre) MP: Años de estudios aprobados $=0-9$ (madre) y $13+$ (padre) mp: Años de estudios aprobados $=0-9$ (madre) y $0-9$ (padre)

Figura 1. Riesgo atribuible de mortalidad infantil de las 4 combinaciones de años de estudios aprobados por la madre y el padre (Chile, 2007).

\section{Discusión}

El presente trabajo demuestra la existencia de una interacción aditiva de tipo sinérgico entre los años de estudios aprobados por ambos progenitores y la tasa de mortalidad infantil, siendo el riesgo atribuible a la coexistencia de un nivel educacional bajo en ambos padres ( $m p)$ es mayor al riesgo esperable de sus efectos individuales $(R A m p>R A m P+R A M p)$.

En Chile, en 1965 se decretó la obligatoriedad y gratuidad de la enseñanza básica ( 8 años), derecho extendido en 2003 a la enseñanza media (4 años). Para el año 2007, la escolaridad promedio de los mayores de 15 años era 10,1 años (mujeres) y 10,4 años (varones), respectivamente. El nivel de educación alcanzado por la población entre 25 y 34 años se distribuía en $1 \%$ sin escolaridad, $8 \%$ enseñanza básica incompleta, 23\% educación básica completa, $42 \%$ educación media completa y $27 \%$ superior completa ${ }^{11}$. En este escenario, y dada la estrecha asociación entre nivel socioeconómico y años de estudios aprobados, es razonable pensar que la combinación $m p$ corresponde a progenitores adolescentes y/o de nivel socioeconómico bajo, ambos reconocidos factores de riesgo para el menor de 1 año, hecho que en la práctica se traduce en un riesgo de muerte 2,7 veces mayor al basal (i.e. riesgo de la combinación $M P$ ).

La constatación de la existencia de una interacción entre el nivel educacional materno y paterno obliga a pronunciarse sobre la influencia que ejerce la figura paterna sobre el pronóstico de salud del menor de 1 año. En 
situaciones en las cuales la madre presenta un nivel educacional bajo $(m)$, la presencia de un padre de nivel educacional alto $(P)$ se asocia a una reducción del riesgo de muerte del menor de 1 año de vida de 2,65 ( $\mathrm{mp})$ a 1,21 ( $\mathrm{mP})$. Estas cifras demuestran la importancia que tiene la figura paterna en la salud del menor de 1 año, siendo su presencia física y/o material en la vida del niño amparada en la legislación chilena por un conjunto de leyes que promueven no solamente el reconocimiento del hijo por parte del padre, sino también su mantención económica. No obstante ello, el hecho de que la combinación $M p$ se asocia a un menor riesgo de muerte del menor de 1 año en comparación a la combinación $m P$ refleja la mayor influencia que tiene la figura materna sobre el pronóstico de salud del menor de 1 año, en comparación a la paterna. Esta situación tiene una explicación sociocultural evidente, al ser históricamente la madre quien asume en forma más directa los cuidados de su hijo (a) en prácticamente todos los niveles socioeconómicos. De cualquier modo, la presencia de al menos un progenitor con educación alta (y presumiblemente de mejor nivel socioeconómico) supliría parcialmente las carencias educacionales y económicas de su pareja, siendo ésta la razón por la cual las combinaciones discordantes $m P$ y $M p$ presentan magnitudes de riesgo ligeramente superiores al riesgo basal $(M P)$.

Internacionalmente, una experiencia similar fue reportada por Bakkteig ${ }^{12}$, quien evaluó la interacción entre los años de estudios aprobados maternos y paternos sobre la mortalidad perinatal y postneonatal en 199596 nacidos vivos únicos y muertes fetales registradas en Noruega en el período 1979-82, desagregando los años de estudios aprobados en las mismas categorías que nuestro estudio. Tomando como referencia la combinación $M P$, la razón de tasas de la combinación $m p$ para mortalidad perinatal fue 1,8 y para mortalidad postneonatal fue 1,5. Si bien en este estudio se logró demostrar el impacto de la educación del padre sobre la mortalidad perinatal y postneonatal, los datos no apoyan la existencia de un fenómeno de interacción aditiva de tipo sinérgico, hecho posiblemente explicado por ciertas características inherentes a su sociedad (e.g. mayor red de apoyo para parejas tipo $\mathrm{mp}$ ). Es importante destacar que en aquel estudio la combinación $m p$ presentó la mayor prevalencia de recién nacidos de bajo peso y el menor promedio de peso de nacimiento de las 9 combinaciones. Por desgracia, el nivel de desagregación de los datos utilizados en nuestro estudio impidió verificar tales asociaciones en nuestra población.

Por último, es importante señalar que nuestro estudio no está exento de limitaciones. Existe una serie de variables materno-fetales y socio-demográficas que cumplen la definición de variables confundentes y que no han sido ajustadas, principalmente por carecer de información para hacerlo o bien por no encontrarse ésta desagregada en los niveles que permitan realizar un ajuste adecuado. Al respecto, siendo los años de estudios aprobados un buen estimador del nivel socioeconómico de las personas, las asociaciones e interacciones demostradas pueden reflejar simplemente diferencias en las condiciones de saneamiento básico y acceso a bienes y servicios por parte de ambos progenitores, sin importar mayormente su grado de instrucción. La conducción de estudios que realicen estos ajustes permitirá comprender de mejor forma este fenómeno multifactorial. Por otro lado, aproximadamente el 15\% de los nacidos vivos y muertes de menores de 1 año no contaban con información sobre el nivel educacional de sus padres, situación que tiene el potencial de sesgar los resultados.

En conclusión, el presente estudio ha mostrado una forma simple de abordar el fenómeno de interacción entre variables y ha contribuido a profundizar el entendimiento sobre la forma en la cual operan estos factores. En palabras de De Irala7," "el concepto de interacción es uno de tipo complejo que debe distinguirse claramente de la confusión, ya que su identificación determinará una actitud radicalmente opuesta por parte del investigador: así como en presencia de confusión el objetivo es eliminar una distorsión de la medida de asociación objeto de la investigación, ante la presencia de interacción el objetivo es describir mejor un fenómeno, una riqueza existente en los datos". La evaluación a mitad de período del cumplimiento de los Objetivos Sanitarios para la Década 2000-2010 reveló que para 2004 la TMI 
se redujo en un $17 \%$ (siendo la meta para 2010 una reducción del $25 \%)^{13}$; por su parte, el riesgo relativo entre quintiles extremos de escolaridad materna aumentó en 15\% al comparar los trienios $1998-2000$ y $2000-2003^{14}$, estando pendiente la evaluación a final de período. Esperamos que la presente investigación motive el estudio de interacciones, siendo el fin último contribuir a la implementación de estrategias de salud pública basadas en evidencia destinadas a reducir aún más la ocurrencia de muertes en menores de 1 año y sus inequidades asociadas.

\section{Referencias}

1.- Jiménez J, Romero MI: Reducing infant mortality in Chile: Success in two phases. Health Affairs 2007; 26: 458-65.

2.- Donoso E: Desigualdad en la mortalidad perinatal entre las comunas de la provincia de Santiago. Rev Chil Obstet Ginecol 2004; 69: 112-7.

3.- Donoso E: Desigualdad en mortalidad infantil entre las comunas de la provincia de Santiago. Rev Med Chile 2004; 132: 461-6.

4.- Hertel-Fernández AW, Giusti AE, Sotelo JM: The Chilean infant mortality decline: improvement for whom? Socioeconomic and geographic inequalities in infant mortality, 1990-2005. Bull World Health Organ 2007; 85: 798-804.

5.- Ministerio de Salud: Objetivos Sanitarios para la Dé- cada 2000-2010. Disponible en http://epi.minsal.cl/epi/ html/elvigia/vigia15.pdf (Consultado el 01/04/10).

6.- Kaempffer AM, Medina E: Mortalidad infantil reciente en Chile: Éxitos y desafíos. Rev Chil Pediatr 2006; 77 : 492-500.

7.- De Irala J, Martínez-González MA, Guillén F: ¿Qué es una variable modificadora de efecto? Med Clin (Barc) 2001; 117: 297-302.

8.- Rothman K, Greenland S, Walker A: Concepts of interaction. Am J Epidemiol 1980; 112: 467-70.

9.- Instituto Nacional de Estadísticas (INE): Anuario de Demografía 2007. Disponible en http://www.ine.cl (Consultado el 02/02/10).

10.- Jewell $N$ : Chapter 10. Interaction. En: Statistics for Epidemiology. 2004 Chapmann \& Hall/CRC.

11.- Ministerio de Educación: Indicadores de la Educación en Chile 2007-2008 (Documento Preliminar). Disponible en http://ded.mineduc.cl (Consultado el 01/04/10).

12.- Bakketeig L, Cnattingius $S$, Knudsen L: Socioeconomic differences in fetal and infant mortality in Scandinavia. J Public Health Policy 1993; 14: 82-90.

13.- Ministerio de Salud: Objetivos Sanitarios para la Década 2000-2010. 'Estado de avance en los objetivos de impacto y desarrollo. Capítulo I: Mejorar los logros sanitarios alcanzados'. Disponible en http://epi.minsal. cl/epi/html/sdesalud/OS/EVOS_cap_I.pdf (Consultado el 01/04/10).

14.- Ministerio de Salud: Objetivos Sanitarios para la Década 2000-2010. 'Evaluación objetivo III: Disminuir las desigualdades en Salud'. Disponible en http://epi.minsal.cl/epi/html/sdesalud/OS/EvOS_III.pdf (Consultado el 01/04/10). 